\title{
The First Data Release of the Sloan Digital Sky Survey
}

\section{Citation}

Abazajian, Kevork, Jennifer K. Adelman-McCarthy, Marcel A. Ageros, Sahar S. Allam, Scott F. Anderson, James Annis, Neta A. Bahcall, et al. 2003. "The First Data Release of the Sloan Digital Sky Survey." The Astronomical Journal 126 (4) (October): 2081-2086. doi:10.1086/378165.

\section{Published Version}

doi:10.1086/378165

\section{Permanent link}

http://nrs.harvard.edu/urn-3:HUL.InstRepos:33370052

\section{Terms of Use}

This article was downloaded from Harvard University's DASH repository, and is made available under the terms and conditions applicable to Other Posted Material, as set forth at http:// nrs.harvard.edu/urn-3:HUL.InstRepos:dash.current.terms-of-use\#LAA

\section{Share Your Story}

The Harvard community has made this article openly available.

Please share how this access benefits you. Submit a story.

Accessibility 


\section{THE FIRST DATA RELEASE OF THE SLOAN DIGITAL SKY SURVEY}

Kevork Abazajian, ${ }^{1}$ Jennifer K. Adelman-McCarthy, ${ }^{1}$ Marcel A. Agüeros, ${ }^{2}$ Sahar S. Allam, ${ }^{1}$ Scott F. Anderson, ${ }^{2}$ James Annis, ${ }^{1}$ Neta A. Bahcall,${ }^{3}$ Ivan K. Baldry, ${ }^{4}$ Steven Bastian, ${ }^{1}$ Andreas Berlind, ${ }^{5}$ Mariangela Bernardi, ${ }^{6}$ Michael R. Blanton,${ }^{7}$ Norman Blythe, ${ }^{8}$ John J. Bochanski, Jr., ${ }^{2}$ William N. Boroski, ${ }^{1}$ Howard Brewington, ${ }^{8}$ John W. Briggs, ${ }^{5}$ J. Brinkmann, ${ }^{8}$ Robert J. Brunner, ${ }^{9}$ Tamás Budavári, ${ }^{4}$ Larry N. Carey, ${ }^{2}$ Michael A. Carr, ${ }^{3}$ Francisco J. Castander, ${ }^{10}$ Kuenley Chiu, ${ }^{4}$ Matthew J. Collinge, ${ }^{3}$ A. J. Connolly, ${ }^{11}$ Kevin R. Covey, ${ }^{2}$ István Csabai, ${ }^{12,4}$ Julianne J. Dalcanton, ${ }^{2}$ Scott Dodelson,${ }^{1,5}$ Mamoru Doi,${ }^{13}$ Feng Dong, ${ }^{3}$ Daniel J. Eisenstein, ${ }^{14}$ Michael L. Evans, ${ }^{2}$ Xiaohui Fan,${ }^{14}$ Paul D. Feldman, ${ }^{4}$ Douglas P. Finkbeiner, ${ }^{3}$ Scott D. Friedman, ${ }^{15}$ Joshua A. Frieman,,${ }^{1,5}$ Masataka Fukugita, ${ }^{16}$ Roy R. Gal,${ }^{4}$ Bruce Gillespie, ${ }^{8}$ Karl Glazebrook, ${ }^{4}$ Carlos F. Gonzalez, ${ }^{1}$ Jim Gray,${ }^{17}$ Eva K. Grebel, ${ }^{18}$ Lauren Grodnicki, ${ }^{5}$ James E. GunN, ${ }^{3}$ ViJay K. Gurbani, ${ }^{1,19}$ Patrick B. Hall, ${ }^{3,20}$ Lei HaO, ${ }^{3}$ Daniel Harbeck, ${ }^{18}$ Frederick H. Harris, ${ }^{21}$ Hugh C. Harris, ${ }^{21}$ Michael Harvanek, ${ }^{8}$ Suzanne L. Hawley, ${ }^{2}$ Timothy M. Heckman, ${ }^{4}$ J. F. Helmboldt,${ }^{22}$ John S. Hendry, ${ }^{1}$ Gregory S. Hennessy ${ }^{23}$ Robert B. Hindsley, ${ }^{24}$ David W. Hogg, ${ }^{7}$ Donald J. Holmgren, ${ }^{1}$ Jon A. Holtzman, ${ }^{22}$ Lee Homer, ${ }^{2}$ Lam Hui, ${ }^{1}$ Shin-ichi Ichikawa, ${ }^{25}$ Takashi IchiKawa, ${ }^{26}$ John P. Inkmann, ${ }^{1}$ ŽElJKo Ivezić, $^{3}$ Sebastian Jester, ${ }^{1}$ David E. Johnston, ${ }^{5}$ Beatrice Jordan, ${ }^{8}$ Wendell P. Jordan, ${ }^{8}$ Anders M. Jorgensen, ${ }^{27}$ Mario Jurić, ${ }^{3}$ Guinevere Kauffmann, ${ }^{28}$ Stephen M. Kent, ${ }^{1}$

S. J. Kleinman, ${ }^{8}$ G. R. Knapp, ${ }^{3}$ Alexei Y. Kniazev, ${ }^{18}$ Richard G. Kron,${ }^{5,1}$ Jurek Krzesiński,,${ }^{8,29}$ Peter Z. Kunszt, ${ }^{4,30}$ Nickolai Kuropatkin, ${ }^{1}$ Donald Q. Lamb,${ }^{5,31}$ Hubert Lampeitl, ${ }^{1}$ Bryan E. Laubscher, ${ }^{27}$ Brian C. Lee, ${ }^{32}$

R. French Leger, ${ }^{1}$ Nolan Li, ${ }^{4}$ Adam Lidz, ${ }^{1}$ Huan Lin, ${ }^{1}$ Yeong-Shang Loh,${ }^{3}$ Daniel C. Long, ${ }^{8}$ Jon Loveday ${ }^{33}$ Robert H. Lupton,${ }^{3}$ Tanu Malik, ${ }^{4}$ Bruce Margon, ${ }^{15}$ Peregrine M. McGehee, ${ }^{22,27}$ Timothy A. McKay, ${ }^{34}$ Avery Meiksin, ${ }^{35}$ Gajus A. Miknaitis, ${ }^{2}$ Bhasker K. Moorthy, ${ }^{22}$ Jeffrey A. Munn, ${ }^{21}$ Tara Murphy,${ }^{35}$ Reiko Nakajima, ${ }^{36}$ Vijay K. Narayanan, ${ }^{3}$ Thomas Nash, ${ }^{1}$ Eric H. Neilsen, Jr., ${ }^{1}$ Heidi Jo Newberg, ${ }^{37}$ Peter R. Newman, ${ }^{8}$ Robert C. Nichol, ${ }^{6}$ Tom Nicinski, ${ }^{1,38}$ Maria Nieto-Santisteban, ${ }^{4}$ Atsuko Nitta,${ }^{8}$ Michael Odenkirchen, ${ }^{18}$ Sadanori Okamura,${ }^{39}$ Jeremiah P. Ostriker, ${ }^{3}$ Russell Owen, ${ }^{2}$ Nikhil Padmanabhan, ${ }^{40}$ John Peoples, ${ }^{1}$ Jeffrey R. Pier, ${ }^{21}$ Bartosz Pindor, ${ }^{3}$

\footnotetext{
${ }^{1}$ Fermi National Accelerator Laboratory, P.O. Box 500, Batavia, IL 60510.

${ }^{2}$ Department of Astronomy, University of Washington, Box 351580, Seattle, WA 98195.

${ }^{3}$ Department of Astrophysical Sciences, Princeton University, Princeton, NJ 08544.

${ }^{4}$ Center for Astrophysical Sciences, Department of Physics and Astronomy, Johns Hopkins University, 3400 North Charles Street, Baltimore, MD 21218-2686.

${ }^{5}$ Department of Astronomy and Astrophysics, University of Chicago, 5640 South Ellis Avenue, Chicago, IL 60637.

${ }^{6}$ Department of Physics, Carnegie Mellon University, Pittsburgh, PA 15213.

${ }^{7}$ Center for Cosmology and Particle Physics, Department of Physics, New York University, 4 Washington Place, New York, NY 10003.

8 Apache Point Observatory, P.O. Box 59, Sunspot, NM 88349.

${ }^{9}$ Department of Astronomy, University of Illinois, 1002 West Green Street, Urbana, IL 61801.

${ }^{10}$ Institut d'Estudis Espacials de Catalunya/CSIC, Gran Capitá 2-4, E-08034 Barcelona, Spain.

${ }^{11}$ Department of Physics and Astronomy, University of Pittsburgh, 3941 O'Hara Street, Pittsburgh, PA 15260.

12 Department of Physics, Eötvös Loránd University, Budapest, Pf. 32, H-1518 Budapest, Hungary.

${ }^{13}$ Institute of Astronomy and Research Center for the Early Universe, School of Science, University of Tokyo, 2-21-1 Osawa, Mitaka, Tokyo 181-0015, Japan.

${ }^{14}$ Steward Observatory, 933 North Cherry Avenue, Tucson, AZ 85721

${ }_{16}^{15}$ Space Telescope Science Institute, 3700 San Martin Drive, Baltimore, MD 21218.

${ }^{16}$ Institute for Cosmic Ray Research, University of Tokyo, 5-1-5 Kashiwa, Kashiwa City, Chiba 277-8582, Japan.

17 Microsoft Research, 455 Market Street, No. 1690, San Francisco, CA 94105.

${ }_{18}$ Max-Planck-Institut für Astronomie, Königstuhl 17, D-69117 Heidelberg, Germany.

${ }^{19}$ Lucent Technologies, 2000 Lucent Lane, Naperville, IL 60566.

${ }^{20}$ Departamento de Astronomía y Astrofísica, Facultad de Física, Pontificia Universidad Católica de Chile, Casilla 306, Santiago 22, Chile.

${ }^{21}$ US Naval Observatory, Flagstaff Station, P.O. Box 1149, Flagstaff, AZ 86002.

${ }_{22}^{2}$ Department of Astronomy, MSC 4500, New Mexico State University, P.O. Box 30001, Department 4500, Las Cruces, NM 88003.

${ }^{23}$ US Naval Observatory, 3540 Massachusetts Avenue, NW, Washington, DC 20392-5420.

${ }^{24}$ Remote Sensing Division, Code 7215, Naval Research Laboratory, 4555 Overlook Avenue, SW, Washington, DC 20375.

${ }^{25}$ National Astronomical Observatory, 2-21-1 Osawa, Mitaka, Tokyo 181-8588, Japan.

${ }^{26}$ Astronomical Institute, Tohoku University, Aramaki, Aoba, Sendai 980-8578, Japan.

${ }^{27}$ Los Alamos National Laboratory, P.O. Box 1663, Los Alamos, NM 87545.

${ }^{28}$ Max-Planck-Institut für Astrophysik, Karl Schwarzschildstrasse 1, D-85748 Garching, Germany.

${ }^{29}$ Obserwatorium Astronomiczne na Suhorze, Akademia Pedagogiczna w Krakowie, ulica Podchorążych 2, PL-30-084 Kraków, Poland.

30 CERN, IT Division, CH-1211 Geneva 23, Switzerland.

${ }^{31}$ Enrico Fermi Institute, University of Chicago, 5640 South Ellis Avenue, Chicago, IL 60637.

32 Lawrence Berkeley National Laboratory, 1 Cyclotron Road, Berkeley, CA 94720-8160.

${ }_{33}$ Astronomy Centre, University of Sussex, Falmer, Brighton BN1 9QJ, UK

${ }^{34}$ Department of Physics, University of Michigan, 500 East University Avenue, Ann Arbor, MI 48109.

${ }^{35}$ Institute for Astronomy, Royal Observatory, University of Edinburg, Blackford Hill, Edinburgh EH9 3HJ, UK.

36 Department of Physics, University of Pennsylvania, Philadelphia, PA 19104.

${ }^{37}$ Department of Physics, Applied Physics, and Astronomy, Rensselaer Polytechnic Institute, 110 Eighth Street, Troy, NY 12180-3590.

${ }^{38}$ Lucent Technologies, 2701 Lucent Lane, Lisle, IL 60532.

39 Department of Astronomy and Research Center for the Early Universe, University of Tokyo, 7-3-1 Hongo, Bunkyo, Tokyo 113-0033, Japan.

40 Joseph Henry Laboratories, Princeton University, Princeton, NJ 08544.
} 
Adrian C. Pope, ${ }^{4}$ Thomas R. Quinn, ${ }^{2}$ R. R. Rafikov, ${ }^{41}$ Sean N. Raymond, ${ }^{2}$ Gordon T. Richards,${ }^{3}$ Michael W. Richmond, ${ }^{42}$ Hans-Walter Rix, ${ }^{18}$ Constance M. Rockosi, ${ }^{2}$ Joop Schaye, ${ }^{41}$ David J. Schlegel, ${ }^{3}$ Donald P. Schneider, ${ }^{43}$ Joshua Schroeder, ${ }^{3}$ Ryan Scranton, ${ }^{11}$ Maki Sekiguchi, ${ }^{16}$ Uroš Seljak, ${ }^{3}$ Gary Sergey, ${ }^{1}$ Branimir Sesar, ${ }^{44}$ Erin Sheldon, ${ }^{5}$ Kazu Shimasaku, ${ }^{39}$ Walter A. Siegmund, ${ }^{45}$ Nicole M. Silvestri, ${ }^{2}$ Allan J. Sinisgalli, ${ }^{3}$ Edwin Sirko, ${ }^{3}$ J. Allyn Smith, ${ }^{46,27}$ Vernesa Smolčić, ${ }^{44}$ Stephanie A. Snedden, ${ }^{8}$ Albert Stebbins, ${ }^{1}$ Charles Steinhardt, ${ }^{3}$ Gregory Stinson, ${ }^{2}$ Chris Stoughton, ${ }^{1}$ Iskra V. Strateva, ${ }^{3}$ Michael A. Strauss, ${ }^{3}$ Mark SubbaRao, ${ }^{5}$ Alexander S. Szalay, ${ }^{4}$ István Szapudi, ${ }^{45}$ Paula Szkody, ${ }^{2}$ Lidia Tasca, ${ }^{28}$ Max Tegmark, ${ }^{36}$ Aniruddha R. Thakar, ${ }^{4}$ Christy Tremonti, ${ }^{4,14}$ Douglas L. Tucker, ${ }^{1}$ Alan Uomoto, ${ }^{4}$ Daniel E. Vanden Berk, ${ }^{11,1}$ Jan Vandenberg, ${ }^{4}$ Michael S. Vogeley, ${ }^{47}$ Wolfgang Voges, ${ }^{48}$ Nicole P. Vogt, ${ }^{22}$ Lucianne M. Walkowicz, ${ }^{2}$ David H. Weinberg, ${ }^{49}$ Andrew A. West, ${ }^{2}$ Simon D. M. White, ${ }^{28}$ Brian C. Wilhite, ${ }^{5}$ Beth Willman, ${ }^{2}$ Yongzhong Xu, ${ }^{36}$ Brian Yanny, ${ }^{1}$ Jean Yarger, ${ }^{8}$ Naoki Yasuda, ${ }^{25}$ Ching-Wa Yip, ${ }^{11}$ D. R. Yocum, ${ }^{1}$ Donald G. York,, 31 Nadia L. Zakamska, ${ }^{3}$ Idit Zehavi, ${ }^{5}$ Wei Zheng, ${ }^{4}$ Stefano Zibetti, ${ }^{28}$ and Daniel B. Zucker ${ }^{18}$

Received 2003 May 27; accepted 2003 July 2

\section{ABSTRACT}

The Sloan Digital Sky Survey (SDSS) has validated and made publicly available its First Data Release. This consists of $2099 \mathrm{deg}^{2}$ of five-band $(u, g, r, i, z)$ imaging data, 186,240 spectra of galaxies, quasars, stars and calibrating blank sky patches selected over $1360 \mathrm{deg}^{2}$ of this area, and tables of measured parameters from these data. The imaging data go to a depth of $r \approx 22.6$ and are photometrically and astrometrically calibrated to $2 \% \mathrm{rms}$ and 100 mas rms per coordinate, respectively. The spectra cover the range $3800-9200 \AA$, with a resolution of 1800-2100. This paper describes the characteristics of the data with emphasis on improvements since the release of commissioning data (the SDSS Early Data Release) and serves as a pointer to extensive published and on-line documentation of the survey.

Key words: atlases — catalogs — surveys

\section{INTRODUCTION}

The Sloan Digital Sky Survey (SDSS) is a photometric and spectroscopic survey, using a dedicated $2.5 \mathrm{~m}$ telescope at Apache Point Observatory in New Mexico, of many thousands of square degrees of high Galactic latitude sky. The scientific goals that define the scope of the project (York et al. 2000) relate to large-scale structure seen in the distribution of galaxies and quasars. In addition to addressing these issues, the survey data products are proving valuable for many other astronomical problems, from asteroids to Galactic structure, from rare types of white dwarf stars to the highest-redshift quasars. The validated data are being released at approximately annual intervals. Each release includes sufficient information to allow statistical analysis, e.g., measures of data quality and the completeness of the source lists. The first SDSS Data Release (DR1) amounts to about $20 \%$ of the total SDSS survey goal.

In summer 2001 the SDSS released the results of observations obtained during the commissioning phase of the SDSS; this Early Data Release (EDR) is described by Stoughton et al. (2002), which contains extensive information on the SDSS data and data processing software..$^{50}$ The purpose of the present paper is to formally mark the first SDSS data release and to provide a quick guide to the contents of the Web site. The sky coverage of the imaging and spectroscopic components of the DR1 are shown in Figure 1.

\section{PUBLISHED DOCUMENTATION}

A number of papers have been published that provide important technical background relevant, but not limited, to DR1. In this section we review these publications.

A technical summary of the project is given by York et al. (2000). This is an introduction to extensive on-line discussion of the hardware (the Project Book). ${ }^{51}$ The imaging camera is described by Gunn et al. (1998).

The Early Data Release is described by Stoughton et al. (2002), which includes an extensive discussion of the data outputs and software. More details of the photometric pipeline may be found in Lupton et al. (2001).

Strauss et al. (2002) give the target selection procedures for the main galaxy sample of the SDSS. This paper provides the basis by which one can construct a statistically complete sample of galaxies with spectra. Eisenstein et al. (2001) describe the procedure for targeting a magnitudeand color-selected sample of luminous red galaxies (LRGs) at redshifts up to $z=0.55$. The redshift histograms of the objects from these two samples in the DR1 are given in Figure 2.

Richards et al. (2002) present the algorithm that is currently being used to target quasars from SDSS photometry, although the DR1 sample (like the EDR sample) uses a more heterogeneous set of algorithms since the DR1 data predate the implementation of this specific algorithm; see Schneider et al. (2003) for more details and a formal catalog

\footnotetext{
${ }^{41}$ School of Natural Sciences, Institute for Advanced Study, Einstein Drive, Princeton, NJ 08540.

42 Department of Physics, Rochester Institute of Technology, 85 Lomb Memorial Drive, Rochester, NY 14623-5603.

${ }^{43}$ Department of Astronomy and Astrophysics, 525 Davey Laboratory, Pennsylvania State University, University Park, PA 16802.

${ }^{44}$ Department of Physics, University of Zagreb, Bijenička cesta 32, HR-10000 Zagreb, Croatia.

45 Institute for Astronomy, 2680 Woodlawn Road, Honolulu, HI 96822.

46 Department of Physics and Astronomy, University of Wyoming, Laramie, WY 82071.

47 Department of Physics, Drexel University, Philadelphia, PA 19104.

48 Max-Planck-Institut für extraterrestrische Physik, Giessenbachstrasse 1, D-85741 Garching, Germany.

49 Department of Astronomy, Ohio State University, Columbus, OH 43210.

${ }^{50}$ A similarly comprehensive description of the DR1 data and derived parameters may be found at http://www.sdss.org/dr1 (hereafter " the Web site").

${ }^{51}$ Available at http://astro.princeton.edu/PBOOK/welcome.htm.
} 

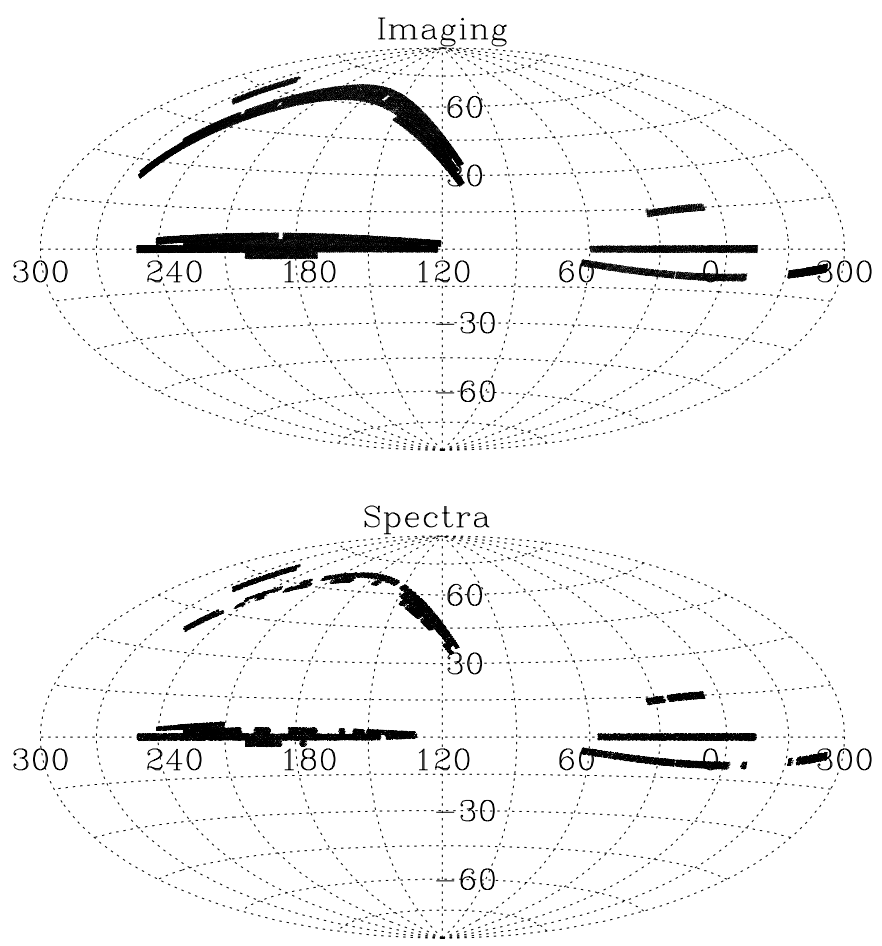

FIG. 1.-Distribution on the sky of the imaging scans and spectroscopic plates included in the DR1. This is an Aitoff projection in equatorial coordinates. The total sky area covered by the imaging is $2099 \mathrm{deg}^{2}$ and by the spectroscopy is $1360 \mathrm{deg}^{2}$.

of DR1 quasars. The redshift histogram of spectroscopically confirmed quasars in the DR1 is given in Figure 3.

These spectroscopic samples are assigned plates and fibers using an algorithm described by Blanton et al. (2003).

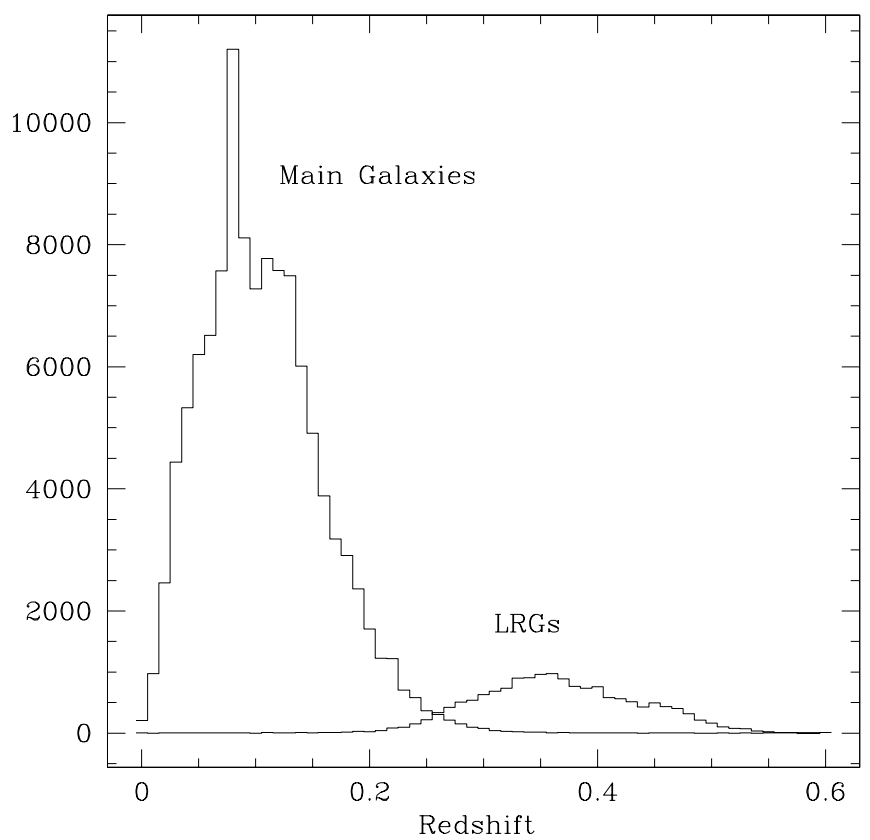

FIG. 2.-Redshift histogram for objects spectroscopically classified as galaxies in DR1. The curve labeled "Main Galaxies" is the flux-limited sample, containing 113,199 galaxies. The curve labeled " LRGs" is a colorselected sample designed to contain intrinsically luminous, red galaxies, containing 15,921 galaxies.

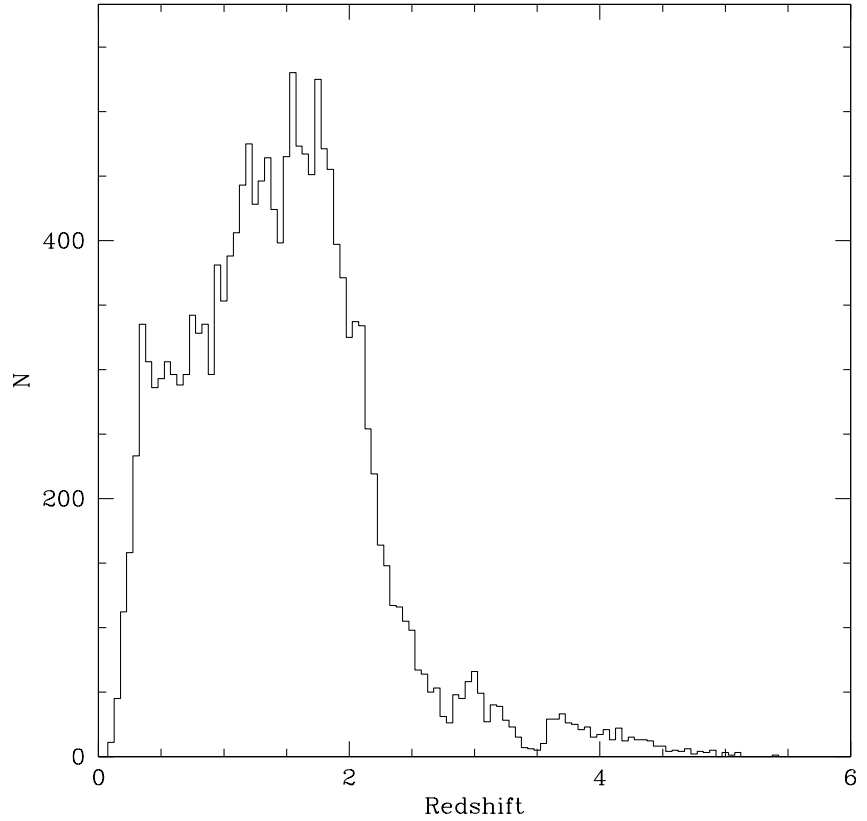

FIG. 3.-Redshift histogram for objects spectroscopically classified as quasars in DR1 (and with luminosities $M_{B}<-22$ ), including 16,847 objects. The catalog of bona fide quasars in DR1 is presented in Schneider et al. (2003).

Pier et al. (2003) describe the methods and algorithms involved in the astrometric calibration of the survey, and present a detailed analysis of the accuracy achieved.

The network of primary photometric standard stars is described by Smith et al. (2002). The photometric system itself is described by Fukugita et al. (1996), and the system which monitors the site photometricity is described by Hogg et al. (2001).

The official (IAU designation) SDSS naming convention for an object is SDSS JHHMMSS.ss \pm DDMMSS.s, where the coordinates are truncated, not rounded. This format should be used at least once for every object listed in a paper using SDSS data.

\section{CONTENTS OF DR1}

The imaging portion of DR 1 comprises $2099 \mathrm{deg}^{2}$ of sky imaged in five wavebands $(u, g, r, i, z)$, containing photometric parameters of 53 million unique objects. Within this area, DR1 includes spectroscopic data (spectra and quantities derived therefrom) for photometrically defined samples of quasars and galaxies, as well as incomplete samples of stars. The spectroscopic data cover $1360 \mathrm{deg}^{2} .52$

SDSS collects imaging data in strips which follow great circles. Two interleaving strips together make up a stripe 2.5 wide; at the equator of the system of great circles, stripes are separated by 2.5 . A continuous scan of a piece of a strip on a particular night is called a run; this is the natural unit of imaging data. Data from 62 runs are included in DR1. The DR1 footprint is defined by all nonrepeating survey-quality runs within the a priori defined elliptical survey area (York et al. 2000) obtained prior to 2001 July 1; in fact, $34 \mathrm{deg}^{2}$ of DR1 imaging data lie outside this ellipse. While the DR1 scans do not repeat a given area of sky, they do overlap to

52 The details of the sky coverage can be found at http://www.sdss.org/ dr1/coverage. 
some extent, and the data in the overlaps are included in DR1 as well.

Spectroscopy is undertaken with guided exposures of overlapping tiles ("plates"), each $3^{\circ}$ in diameter. For each plate 640 spectroscopic fibers are available. DR 1 consists of 291 plates, the centers of which lie within the boundaries defined by the DR1 imaging footprint.

The surface density of spectroscopic targets per square degree consists, on average, of roughly 90 galaxies in a fluxlimited sample, an additional 12 galaxies of a flux- and color-limited sample of luminous red galaxies (LRGs), and 18 quasar candidates. Each plate is assigned 18 calibration stars and 32 fibers on blank sky for sky subtraction. Finally, extra fibers available in a given region of sky are assigned to objects matching ROSAT (X-ray; Voges et al. 1999) and FIRST (radio; Becker, White, \& Helfand 1995) sources, as well as unusual stars of various types. The spectroscopic targeting of all of these samples is based on the photometric quantities produced by the SDSS pipelines.

DR1 includes the footprint of the sky already released in the EDR. All of the EDR data have been reprocessed with the latest versions of the SDSS software. In some parts of the sky, better data (both imaging and spectroscopy) have been substituted for the older, commissioning data of the EDR.

The data products in DR1 include the following:

1. Images.-The "corrected frames" (flat-fielded, skysubtracted, and calibrated subimages corrected for bad columns, bleed trails, and cosmic rays, each $13 ! 6 \times 9^{\prime}$ ) in five bands, available in both FITS and JPEG format; a mask file that records how each pixel was used in the imaging dataprocessing pipelines; $4 \times 4$ binned images (i.e., with 1". 6 pixels) of the corrected frames after detected objects have been removed; and "atlas images" (cutouts from the corrected frames of each detected object).

2. Image parameters.-The positions, fluxes, shapes, and errors thereof for all detected objects in the images, as well as information about how these objects were spectroscopically targeted.

3. Spectra.-The flux- and wavelength-calibrated, skysubtracted spectra, with error and mask arrays, and the resolution of the spectra as a function of wavelength.

4. Spectroscopic parameters.-The redshift and spectral classification of each object with a spectrum, as well as the properties of detected emission lines and various further spectral indices.

5. Other data products.-Astrometric and photometric calibration files, the point spread function of the images, GIF and postscript plots of spectra, and "finding charts" (cutouts of the survey image area according to specified limits in right ascension and declination) in a number of formats.

These DR1 data products are available at the Web site, which includes detailed description of the data, and documentation of the access tools.

\section{CHANGES IN DR1 WITH RESPECT TO THE EARLY DATA RELEASE}

The description of the SDSS data, file structures, and processing pipelines presented in Stoughton et al. (2002) remains an essential point of departure for understanding and using the data products in the First Data Release. How- ever, there have been some significant changes in the data processing since the EDR; we comment briefly here on some of the more important ones.

1. The photometric equations have been reformulated to be in the natural system of the $2.5 \mathrm{~m}$ telescope, making the relation between measured counts and magnitude a simple one. The mean colors of stars on the old and new systems have been forced to be the same. The changes from previously published photometry due to this are subtle, typically no more than few hundredths of a magnitude. To distinguish between photometric systems, the new one $(u, g, r, i, z)$ is unadorned, whereas the EDR system was designated with asterisks $\left(u^{*}, g^{*}, r^{*}, i^{*}, z^{*}\right)$. The prime system discussed by Fukugita et al. (1996; $\left.u^{\prime}, g^{\prime}, r^{\prime}, i^{\prime}, z^{\prime}\right)$ now refers only to the native system of the US Naval Observatory Flagstaff Station $1 \mathrm{~m}$ telescope (see Smith et al. 2002), and should not be used in referring to the data from the 2.5 $\mathrm{m}$. As before, all magnitude zero points are approximately (i.e., within $10 \%$ ) on an AB system. The magnitude scale is not exactly logarithmic, but uses an asinh scaling (Lupton, Gunn, \& Szalay 1999; see the Web site for further details). Surface brightnesses, however, are reported on a linear flux scale of "maggies"; 1 maggie corresponds to the surface brightness of a zeroth-magnitude object in $1 \operatorname{arcsec}^{2}$. A surface brightness of 20th mag in $1 \operatorname{arcsec}^{2}$ is therefore $10^{-8}$ maggies.

2. In the EDR, scattered light produced systematic errors in the derived flat field, and therefore in the photometry, especially in the $u$ band. The imaging flat fields have now been corrected for this effect, reducing a major source of systematic error.

3. The EDR version of the photometric pipelines had difficulty following rapid variations in the point-spread function. The DR1 code is more robust to this problem, and has greatly reduced the effects of variable seeing on the photometric measurements.

4. There is a small but measurable nonlinearity in the response of the photometric CCDs, measuring several percent at saturation. This effect has been corrected in the DR1 processing.

5. The EDR image deblender often shredded galaxies with substructure into several individual objects, especially for objects brighter than $r \sim 15$ mag. This behavior has been suppressed, and the vast majority of bright galaxies are now treated properly by the deblender.

6. In addition to the object shape measures of the EDR, the photometric pipeline now calculates so-called adaptive moments (see Bernstein \& Jarvis 2002) that are designed for weak-lensing measurements of faint objects.

7. Cosmic rays are recognized as such by their sharp gradients relative to the point-spread function. An enhanced routine described in Fan et al. (2001) is now implemented as part of the pipeline. This routine sets a flag, MAYBE_CR, which is valuable for assessing the reality of objects detected in only a single band.

8. In the EDR the exponential (Freeman 1970) and de Vaucouleurs (1948) profile models for galaxy images were fit only to the central $3^{\prime \prime}$ radius of each object. This procedure tended to give misleading results for galaxies with large angular extent. The DR1 version of the code does a much more reasonable fit to large galaxies. However, an error was found following the completion of DR1 processing, which causes the model magnitudes to be systematically underesti- 
mated by $0.2 \mathrm{mag}$ (i.e., the model magnitudes are too bright) for galaxies brighter than 20th magnitude. Similarly, the measured radii are systematically too large. This error is the reason that the Web site at the time of publication of the current paper makes reference to a "beta" version of the reduction pipelines. This error will be corrected in future SDSS data releases. Note that this error only affects model magnitudes of galaxies; all other photometry is unaffected by this error. In addition, model colors are essentially unchanged.

9. The astrometric pipeline now uses centroids corrected for asymmetries in the point-spread function, and includes a better treatment of chromatic aberration.

10. The spectroscopic pipeline has much improved flatfielding, bias subtraction, and handling of bad columns and pixels. Sky subtraction has been improved, especially in the red, by allowing for the gradient in the sky brightness across a spectroscopic plate. The spectrophotometric flux calibration is improved as well, as is the correction for absorption lines from the Earth's atmosphere.

11. There have been upgrades to the continuum and linefitting routines in the spectroscopic pipeline. More extensive stellar templates have increased the accuracy of the classification of unusual types of stars.

12. The galaxy spectral-classification eigentemplates for DR1 are created from a much larger sample of spectra $(200,000)$ than were used for the EDR.

\section{DATA QUALITY}

\subsection{Quality of Imaging Data}

The imaging survey is undertaken in photometric conditions (as determined by an all-sky $10 \mu \mathrm{m}$ camera) with no moon. We also impose a nominal limit on the effective width of the point-spread function of 1 !'7 in the $r$ filter. This width is the full width at half maximum of the Gaussian with effective area equal to that of the actual point spread function (PSF) at the center of each frame; it is therefore somewhat larger than the actual full width at half maximum of the PSF, due to the presence of extended low-amplitude wings on the PSF. In the off-line processing, data are declared not to be of survey quality if the width of the point-spread function exceeds this value for an interval longer than about 10 minutes, or if the point-spread function is seen to be rapidly varying, in which case an attempt is made to scan that interval again. The upper panel of Figure 4 shows the cumulative distribution of the width of the point-spread function in DR1 as determined on a frame-by-frame basis; only a very small fraction of the data in DR1 exceeds the seeing threshold. The five filters yield different distributions both because of the dependence of seeing on wavelength, and because the separate filters sample distinct regions of the focal plane.

The bottom panel of Figure 4 shows the distribution of sky brightness values in DR1, averaging over each frame. This value has been corrected for atmospheric extinction to zero air mass, and therefore is biased to higher brightness, by 0.65 mags in $u$, but only 0.08 mag in $z$. The sky brightness, together with the instrumental throughput and the atmospheric extinction and air mass, allow one to compute the expected signal-to-noise ratio for the image of an object of known brightness and profile; the sensitivity curves for the five bands are available on the Web site..$^{53}$

\footnotetext{
${ }^{53}$ See http://www.sdss.org/dr1/instruments/imager.
}
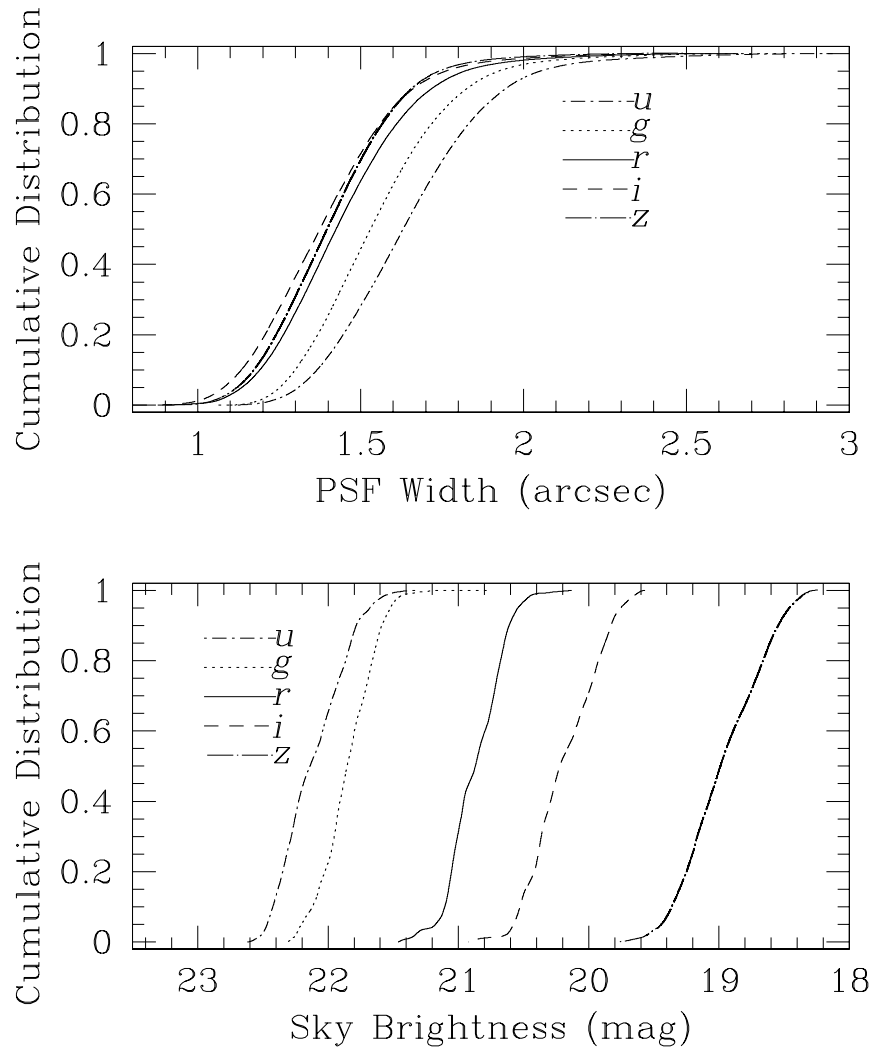

FIG. 4.-Top: Cumulative distribution of values of the seeing in arcseconds for all frames in DR 1 in each of the five filters. Note that over $90 \%$ of the survey data meet the nominal specification of seeing better than 1"7 in $r$. Bottom: Cumulative distribution of the values of the sky brightness in units of magnitudes per square arcsecond for all frames in DR1, as measured in the five filters.

The photometric zero point is transferred from stars calibrated for this purpose using an auxiliary photometric telescope, in $27^{\prime} \times 27^{\prime}$ regions distributed approximately every $15^{\circ}$ along each stripe. A number of tests allow us to quantify the uniformity of the photometric zero points and the accuracy of the calibrations:

1. repeatability of photometry in regions of sky in which runs overlap (see Ivezić et al. 2003);

2. constancy of the locus of stars in color-color space;

3. lack of structure in the stellar or galaxy distribution on the sky correlated with run geometry, seeing, foreground reddening, and sky brightness;

4. comparison of SDSS photometry with externally calibrated standard star fields.

From these results, the photometric zero point varies across the DR 1 footprint by less than 0.02 mag rms in the $r$ band, $0.02 \mathrm{mag}$ rms in the colors $g-r$ and $r-i$, and $0.03 \mathrm{mag} \mathrm{rms}$ in the colors $u-g$ and $i-z$.

We can similarly check the astrometric precision; repeat scans confirm that our rms errors are rarely worse than 100 mas per coordinate; a more typical number is 60 mas. See Pier et al. (2003) for an extensive discussion of the astrometric accuracy.

The depth of the imaging data is a function of sky brightness and seeing, but comparisons with deeper fields from the COMBO-17 survey (Wolf et al. 2003) give 50\% completeness for stellar sources at $(u, g, r, i, z)=(22.5,23.2,22.6$, $21.9,20.8)$ under typical conditions. Star-galaxy separation is better than $90 \%$ reliable to $r=21.6$. 


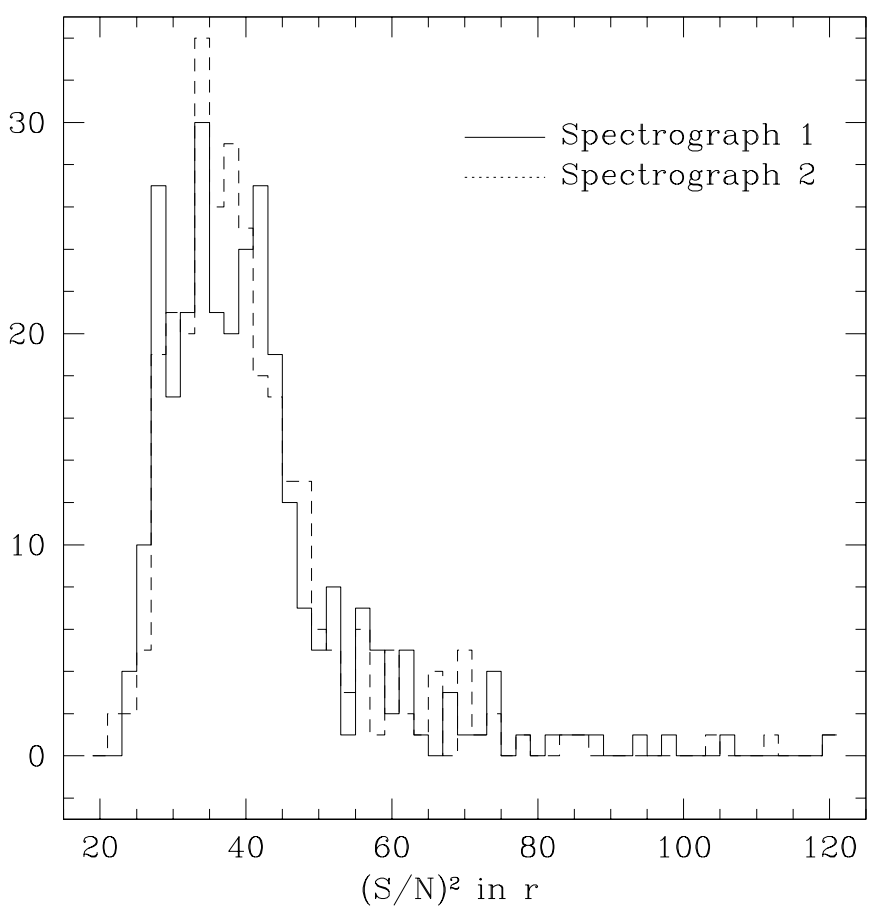

FIG. 5.-Distribution of the values of the square of the spectroscopic signal-to-noise ratio per pixel for an object with $r=20.25$ through a fiber for the 291 plates in DR1. The two spectrographs (each with 320 fibers) are shown separately. All the DR1 plates have $(\mathrm{S} / \mathrm{N})^{2}>20$; note the presence of a number of plates with signal-to-noise ratio much higher than the minimum value.

\subsection{Quality of Spectroscopic Data}

The spectroscopic survey is undertaken in observing conditions that are not photometric, or with seeing worse than 1".7 FWHM, or with some moonlight. A spectroscopic observation is declared to be of survey quality when the mean square of the signal-to-noise ratio per pixel over the spectrum is greater than 15 for objects with fiber magnitudes of $g=20.2, r=20.25$, and $i=19.9$; see the Web site for more details. Figure 5 presents the distribution of the square of the signal-to-noise ratio per pixel for the 291 plates in DR1, for objects with a fiber magnitude $r=20.25$. All the plates clearly exceed the threshold of $(\mathrm{S} / \mathrm{N})^{2}=15$; note the presence of several plates with signal-to-noise ratio exceeding the minimum requirements by factors approaching 3 , as some plates were observed for substantially longer times. The sky subtraction is sufficiently accurate that the noise is close to the photon shot noise.
The FWHM of an unresolved emission line in the spectra is typically 2.5 pixels ( 1 pixel $\sim 65 \mathrm{~km} \mathrm{~s}^{-1}$ ). From repeat observations of galaxies near the survey limit, the redshift accuracy is known to be better than $30 \mathrm{~km} \mathrm{~s}^{-1}$; for bright stars, the redshift accuracy may be better than $10 \mathrm{~km} \mathrm{~s}^{-1}$.

The redshifts and classifications have been checked and updated by comparing results from two independent codes. Roughly $1 \%$ of the spectra (other than the 32 sky spectra per plate) are of low enough signal-to-noise ratio as to be unclassifiable; of the remaining, the error rate is below half a percent.

Data quality also depends on the precision and uniformity with which classes of spectroscopic targets have been selected and observed. The user should be aware that the magnitude limit for the main galaxy sample has ranged from 17.5 to 17.77 in extinction-corrected Petrosian $r$ magnitude through the period covered by DR1. Similarly, the quasar target selection algorithm described in Richards et al. (2002) is a modification of that used in DR 1 ; the newer version is more complete in high-redshift quasars. See the DR1 Web site and the papers cited in $\S 2$ for more details.

As the name implies, DR1 is the first of a series of five releases of what will eventually be the entire Sloan Digital Sky Survey. DR 1 consists of roughly $20 \%$ of the eventual SDSS database. The second data release, DR2, is planned for early 2004. DR2 will increase the total amount of data by $50 \%$ with respect to DR 1 , and it will include a reprocessing of DR1, which will fix the model magnitude bug mentioned in $\S 4$.

Funding for the creation and distribution of the SDSS Archive has been provided by the Alfred P. Sloan Foundation, the Participating Institutions, the National Aeronautics and Space Administration, the National Science Foundation, the Department of Energy, the Japanese Monbukagakusho, and the Max Planck Society. The SDSS Web site is http://www.sdss.org.

The SDSS is managed by the Astrophysical Research Consortium (ARC) for the Participating Institutions. The Participating Institutions are the University of Chicago, Fermilab, the Institute for Advanced Study, the Japan Participation Group, the Johns Hopkins University, Los Alamos National Laboratory, the Max-Planck-Institut für Astronomie, the Max-Planck-Institut für Astrophysik, New Mexico State University, University of Pittsburgh, Princeton University, the United States Naval Observatory, and the University of Washington.
Becker, R. H., White, R. L., \& Helfand, D. J. 1995, ApJ, 450, 559 Bernstein, G. M., \& Jarvis, M. 2002, AJ, 123, 583

Blanton, M. R., Lin, H., Lupton, R. H., Maley, F. M., Young, N., Zehavi, I., \& Loveday, J. 2003, AJ, 125, 2276

de Vaucouleurs, G. 1948, Ann. d'Astrophys., 11, 247

Eisenstein, D. J., et al. 2001, AJ, 122, 2267

Fan, X., et al. 2001, AJ, 122, 2833

Freeman, K. C. 1970, ApJ, 160, 811

Fukugita, M., Ichikawa, T., Gunn, J. E., Doi, M., Shimasaku, K., \& Schneider, D. P. 1996, AJ, 111, 1748

Gunn, J. E., et al. 1998, AJ, 116, 3040

Hogg, D. W., Finkbeiner, D. P., Schlegel, D. J., \& Gunn, J. E. 2001, AJ, 122,2129

Ivezić, Ž., et al. 2003, preprint (astro-ph/0301400)

Lupton, R. H., Gunn, J. E., Ivezić, Z., Knapp, G. R., Kent, S., \& Yasuda N. 2001, in ASP Conf. Proc. 238, Astronomical Data Analysis Software and Systems X, ed. F. R. Harnden, Jr., F. A. Primini, \& H. E. Payne (San Francisco: ASP), 269

\section{REFERENCES}

Lupton, R. H., Gunn, J. E., \& Szalay, A. S. 1999, AJ, 118, 1406

Pier, J. R., Munn, J. A., Hindsley, R. B., Hennessy, G. S., Kent, S. M., Lupton, R. H., \& Ivezić, Z. 2003, AJ, 125, 1559

Richards, G. T., et al. 2002, AJ, 123, 2945

Schneider, D. P., et al. 2003, AJ, submitted

Smith, J. A., et al. 2002, AJ, 123, 2121

Stoughton, C., et al. 2002, AJ, 123, 485

Strauss, M. A., et al. 2002, AJ, 124, 1810

Voges, W., et al. 1999, A\&A, 349, 389

Wolf, C., Meisenheimer, K., Rix, H.-W., Borch, A., Dye, S., \& Kleinheinrich, M. 2003, A\&A, 401, 73

York, D. G., et al. 2000, AJ, 120, 1579 